\title{
GMR
}

\section{Manipulation of components that control feeding behavior in Drosophila melanogaster increases sensitivity to amino acid starvation}

\author{
J.D. Slade and B.E. Staveley \\ Department of Biology, Elizabeth Avenue, Science Building, \\ Memorial University of Newfoundland, St. John's, Newfoundland and Labrador, Canada \\ Corresponding author: B.E. Staveley \\ E-mail: bestave@mun.ca \\ Genet. Mol. Res. 15 (1): gmr.15017489 \\ Received August 20, 2015 \\ Accepted November 9, 2015 \\ Published February 5, 2016 \\ DOI http://dx.doi.org/10.4238/gmr.15017489
}

ABSTRACT. Feeding is a complex behavior that is regulated by several internal mechanisms. Neuropeptides are able to survey quantities of stored energy and inform the organism if nutrient intake is required. In addition to this homeostatic regulation, a post-feeding reward system positively reinforces feeding. Slight adjustments to either system can tilt the balance to affect the energy reserves and survivorship in times of nutrient adversity. Neuropeptide F (NPF), a homolog of the mammalian neuropeptide $\mathrm{Y}$, acts to induce feeding within the homeostatic regulation of this behavior. Drosophila and other insects bear a shorter form of NPF known as short NPF (sNPF) that can influence feeding. A neural hormone regulator, the dopamine transporter (DAT), works to clear dopamine from the synapses. This action may manipulate the post-feeding reward circuit in that lowered dopamine levels depress feeding, and excess dopamine levels encourage feeding. Here, we have overexpressed and impaired the activities of NPF, sNPF, and DAT in Drosophila, and we examined their ability to survive during conditions of amino acid starvation. Too much or too little NPF or sNPF, which are key players in homeostatic feeding regulation, leads to increased sensitivity to amino acid starvation and diminished survivorship 
when compared to controls. When DAT, a member of the post-feeding reward system, is either overexpressed or reduced via mutation, Drosophila has increased sensitivity to amino acid starvation. Taken together, these results indicate that subtle variation in the expression of key components of these systems impacts survivorship during adverse nutrient conditions.

Key words: Starvation; Drosophila; NPF; sNPF; DAT

\section{INTRODUCTION}

The act of feeding by any organism must be modified based on the environment and available nutrition in order to maintain an appropriate energy balance. A disruption in this balance may lead to metabolic diseases, including obesity and eating disorders such as anorexia (Nielsen, 2001; Fairburn and Harrison, 2003). A myriad of genes helps maintain this balance by prompting foraging and feeding, or by encouraging the cessation of feeding. Expression of these genes is often triggered by internal mechanisms that monitor the levels of peripheral energy stores. When there is sufficient energy stored, anorexic genes can signal satiety to cause the organism to stop feeding. When internal energy stores are lowered, the orexic genes can signal to the organism that feeding is necessary to restore energy. Typically this homeostatic process, which in mammals measures energy stores and relays signals between the hypothalamus and the periphery (Hoebel, 1971), works efficiently. However, in an environment where nutrients are readily available, an additional post-satiation reward system can regulate feeding patterns. Although the homeostatic response works well when animals are required to forage, the post-satiation reward can override the homeostatic signals when there is an excess of food (Palmiter, 2007). Therefore, it is important to consider both means of control when evaluating the role of feeding behavior to understand eating disorders.

Drosophila melanogaster is a simple model organism that can be utilized to uncover the potential mechanisms of metabolic disease. Drosophila organ systems include members that function analogously to those in vertebrates. For example, the fat body in Drosophila is utilized to store triglycerides, and this is akin to liver function in vertebrates (Owusu-Ansah and Perrimon, 2014). Drosophila contains a specialized group of cells, the oenocytes, which function like the hepatocytes in vertebrates, which mobilize stored lipids during periods of low food intake. In addition, Drosophila possesses homologous hormones that regulate feeding behavior. These similarities allow Drosophila experiments that are applicable to human health research.

In vertebrates, the neurotransmitter neuropeptide Y (NPY) has been studied extensively as a central enhancer in the control of food intake. NPY is a peptide hormone consisting of 36 amino acids, beginning and ending with a tyrosine $(\mathrm{Y})$ residue, thus giving it its name (Arora and Anubhuti, 2006). First identified in the tapeworm Moniezia expansa (Maule et al., 1991), invertebrate homologs of NPY have been found in major phyla (Maule et al., 1995; de Jong-Brink et al., 2001). However, these homologs encode a 36 amino acid neuropeptide F (NPF), which has a conserved phenylalanine (F) residue at the carboxyl terminus. NPF has been consistently detected in the invertebrate brain, and it is often expressed in the midgut, which points to its role in feeding regulation. Receptors resembling vertebrate NPY receptors have been identified in a number of invertebrates, including Caenorhabditis elegans and D. melanogaster (de Bono and Bargmann, 1998; Garczynski et al., 2002). The structure of the NPF receptor resembles that of 
vertebrate receptors, and the NPF receptor from Drosophila was shown to cross-react with human NPY, suggesting the functional conservation between NPF and NPY (Garczynski et al., 2002). Similar to vertebrates, expression of invertebrate NPF receptors is generally localized to both the central and peripheral nervous systems.

Interestingly, the initial identification of NPF peptides in insects revealed shorter peptides containing only 8 to 10 amino acids. Both long (36 amino acids) and short versions of NPF peptides were found in Drosophila (Vanden Broeck, 2001). The short versions of NPF exhibited similarity to the carboxyl-terminus of the longer version, and were subsequently named short NPFs (sNPFs). Long NPF peptides are considered the functional homologs of vertebrate NPYs, and sNPFs are believed to be independent because each has its own distinct precursor genes and receptors. There are four known sNPF sequences in Drosophila, which are encoded by a single transcription unit (Vanden Broeck, 2001). Analysis of these small peptides in Drosophila indicated that they are expressed in a large number of neurons in both the brain and the stomatogastric system, and they are often co-expressed with other neuropeptides such as insulin and other neurotransmitters (Nassel and Wegener, 2011). Drosophila studies that either reduced sNPF expression or overexpressed the peptide confirmed that it controls the initiation or cessation of feeding. While both peptides appear to affect the control of feeding behavior, only sNPFs are linked to the control of growth.

The dopamine transporter (DAT) is a membrane-bound protein that is central to the regulation of extracellular dopamine levels, which are controlled by the rapid reuptake of the neurotransmitter that acts to clear the synapses. The dopamine neurotransmitter functions in reward-motivated behaviors; thus, changes to extracellular dopamine levels can result in a number of diseases (Palmiter, 2007; Yamamoto and Seto, 2014). Low levels of dopamine are associated with attention deficit hyperactivity disorder (ADHD), depression, and under-eating (Cannon et al., 2004; Sotak et al., 2005). Studies in rats showed that upon feeding or drinking, there was a rapid firing of dopamine in the brain. However, animals with lowered or no dopamine activity were hypoactive, apparently apathetic, and prone to death from starvation or dehydration (Schultz, 2006). Conversely, hyper-dopaminergic mice overfed and eventually became obese (Pecina et al., 2003; Cagniard et al., 2006). Therefore, manipulation of the transporter that regulates the strength and duration of dopamine signaling could lead to similar outcomes. The Drosophila DAT gene was re-isolated in a screen for genes that alter sleep (Kume et al., 2005). A DAT mutant, named fumin (fmn) (Japanese for sleepless), is active for nearly 24 hours in a day. Drosophila DAT expression patterns were concentrated in known dopaminergic neuron locations within larvae and the adult head. Overall, it is evident that dopamine activity could be an effective regulator of feeding.

The prevalence of eating disorders and obesity in modern society has generated a great deal of interest in elucidating the pathways and mechanisms that control feeding behavior. A better understanding of how these systems work to control feeding could lend itself to the enhancement of survival during conditions of nutritional adversity. We investigated the impact on Drosophila survival during amino acid starvation with NPF, sNPF, and DAT overexpression and loss-of-function.

\section{MATERIAL AND METHODS}

\section{Drosophila stocks and culture}

The UAS-NPF and NPF-Gal4 lines (Wu et al., 2003) were received from the laboratory of Dr. P. Shen (University of Georgia), and the UAS-sNPF and UAS-sNPF-RNAi lines (Lee et al., 
2004) were received from the laboratory of Dr. K. Yu (Korea Research Institute of Bioscience and Biotechnology). The DAT mutant line, fmn (Kume et al., 2005), and a control subset line of the standard $w^{1118}$ stock (selected because of lowered activity) were obtained from the laboratory of Dr. Rob Jackson (Tufts University, Boston). The Bloomington Stock Center (Indiana University) supplied the UAS-DAT line, the pan-neural expressing elav-Gal4 transgene, and the responding lines (UAS-GFP, UAS-lacZ, and UAS-rpr). All stocks were maintained on standard corn-meal/ yeast/molasses/agar media in plastic culture vials.

\section{Amino acid starvation ageing assays}

All crosses were performed on standard media, and conditions were maintained at $25^{\circ} \mathrm{C}$ until eclosion. Adult male flies of the critical class were collected daily and placed on amino acid starvation media in groups of 20 or less. Amino acid starvation media consisted of $5 \%$ sucrose in PBS and $3 \%$ agar in plastic culture vials. Flies were assessed daily for the presence of any dead flies until no flies remained, and media were replenished as necessary. Data were analyzed using GraphPad Prism version 5.0 (GraphPad Software, Inc., San Diego, CA, USA), and log-rank tests were conducted.

\section{RESULTS}

\section{Manipulation of NPF expression reduced survivorship on amino acid starvation media}

To reduce the presence of NPF in Drosophila, the experimental approach used a Gal4 transgene with expression that was specific to neurons that produced the neuropeptide. This "driving transgene" was used to overexpress the benign control protein GFP ( $N=353$ males observed), which resulted in a median survivorship of 30 days when aged on the nutrient-deprived media (Table 1, Figure 1A). In comparison, when the NPF-Gal4 driver was used to overexpress a cell death protein reaper ( $r p r, \mathrm{~N}=371$ males observed), the ablation of NPF producing neurons resulted in a median survivorship of 24 days (Table 1, Figure 1A). This difference of six days indicated significant sensitivity to amino acid starvation.

Table 1. Median day of survivorship and total number of observed Drosophila deaths associated with NPF overexpression or loss of function.

\begin{tabular}{l|l|c|c}
\hline Genotype & Condition & Number of deaths observed & Median day of survivorship \\
\hline w; elav-Gal4/UAS-GFP & Control & 330 & 32 \\
\hline w; elav-Gal4/UAS-NPF & Over-expression & 290 & 23 \\
\hline NPF-Gal4/UAS-GFP & Control & 353 & 30 \\
\hline NPF-Gal4/UAS-rpr & Cell death-mediated loss of function & 371 & 24 \\
\hline
\end{tabular}

Overexpression of NPF was achieved via the pan nervous system driver elav-Gal4. The combination of this transgene and the benign responding transgene, UAS-GFP, as a control $(\mathrm{N}=$ 330 males observed) resulted in a median survivorship of 32 days when aged on the amino acid starvation media (Table 1, Figure 1B), which was comparable to the control described above. When elav-Gal4 was used to overexpress NPF in elav-Gal4/UAS-NPF flies ( $N=290$ males observed), the median length of survivorship decreased to 23 days (Table 1, Figure 1B). This difference of seven days indicated significant sensitivity to the amino acid starvation media. 
A

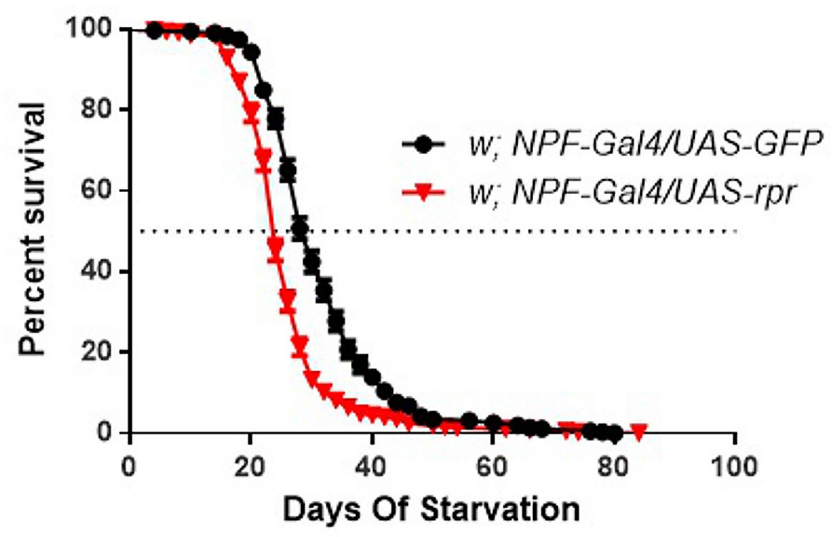

B

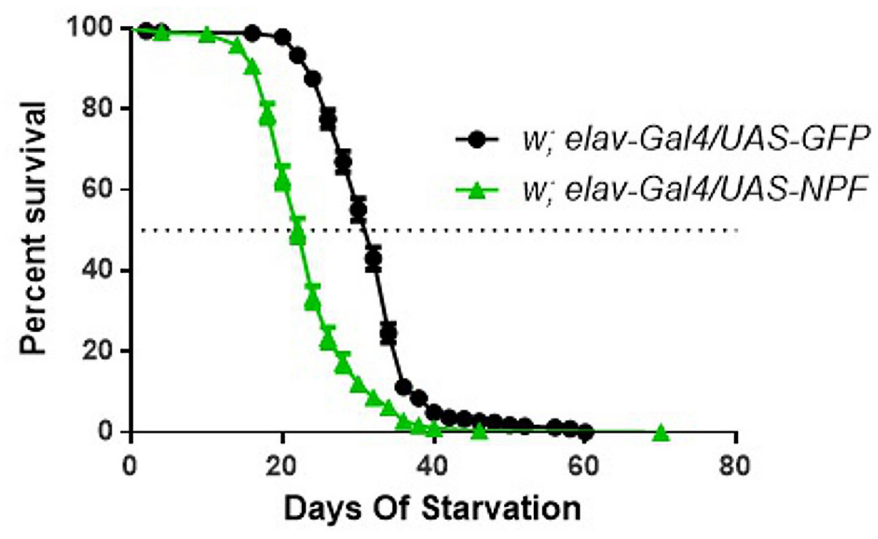

Figure 1. Drosophila survivorship is reduced with either excess or reduced NPF expression during amino acid starvation. A. Cell death-mediated loss of NPF function. Genotypes: w; UAS-GFP/+; NPF-Gal4/+ $(\mathrm{N}=353)$, w; UAS$r p r /+; N P F-G a l 4 /+(N=371)$. B. Over-expression of NPF. Genotypes: w; elav-Gal4/UAS-GFP $(\mathrm{N}=330)$, w; elav-Gal4/ UAS-NPF $(N=290)$. Survivorship is shown as percent survival $(P<0.05$ as determined by log rank). The dotted line represents the median survival of the flies. Error bars represent the standard error of the mean.

\section{Manipulation of sNPF expression increased sensitivity to amino acid starvation}

The nervous system-specific driving transgene, elav-Gal4, was used to either inhibit or increase sNPF expression. As a control ( $N=330$ males observed), this transgene was combined with the benign responder transgene, UAS-GFP, which resulted in a median survivorship of 32 days when aged on amino acid starvation media (Table 2, Figure 2A). To inhibit the expression of $s N P F$, the elav-Gal4 driver was combined with UAS-sNPF-RNAi ( $\mathrm{N}=310$ males observed). Ageing of these flies on amino acid starvation media led to a median survivorship of 22 days (Table 2, Figure 2A), which was a significant reduction of 10 days compared to the control.

To enhance the expression of SNPF, the elav-Gal4 transgene was combined with UASsNPF ( $\mathrm{N}=280$ males observed). These flies exhibited a median survival time of 24 days (Table 2 , Figure $2 \mathrm{~B}$ ), which was a significant reduction of eight days compared to the control. 
Table 2. Median day of survivorship and the total number of observed Drosophila deaths associated with sNPF over-expression or loss of function.

\begin{tabular}{l|l|c|r}
\hline Genotype & Condition & Number of deaths observed & Median day of survivorship \\
\hline w; elav-Gal4/UAS-GFP & Control & 330 & 32 \\
\hline w; elav-Gal4/UAS-sNPF & Over-expression & 280 & 24 \\
\hline w; elav-Gal4/UAS-sNPF-RNAi & RNA interference-mediated loss of function & 310 & 22 \\
\hline
\end{tabular}

$\mathbf{A}$

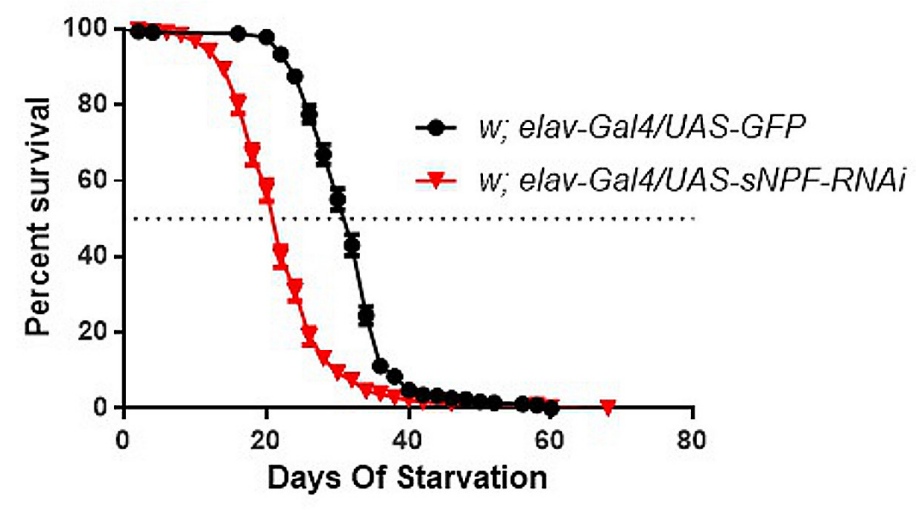

B

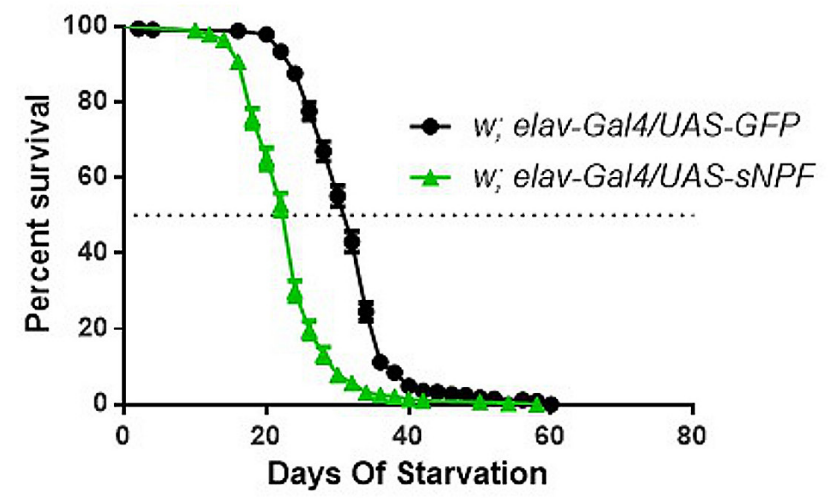

Figure 2. Drosophila survivorship is reduced with either excess or reduced $S N P F$ expression during amino acid starvation. A. RNA interference-mediated loss of sNPF function. Genotypes: w; elav-Gal4/UAS-GFP $(N=330)$, w; elav-Gal4/UAS-sNPF Ri $(\mathrm{N}=310)$. B. Over-expression of sNPF. Genotypes: w; elav-Gal4/UAS-GFP $(\mathrm{N}=330)$, w; elav-Gal4/UAS-sNPF $(\mathrm{N}=280)$. Survivorship is shown as percent survival $(P<0.05$ as determined by log rank). The dotted line represents the median survival of the flies. Error bars represent the standard error of the mean.

\section{Manipulation of DAT expression decreased survivorship on amino acid starvation media}

Reduction in DAT expression was observed in the fmn mutant that was produced by the insertion of an $\sim 2 \mathrm{~kb}$ transposon within $D A T$, and this mutant was found to have little to no DAT activity (Kume et al., 2005). The control for this experiment, a $w^{1118}$ line, had a genetic background into which the fmn mutation was recombined ( $N=508$ males observed). These flies exhibited a 
median survival time of 24 days when challenged with the amino acid starvation media (Table 3 , Figure $3 A$ ). In comparison, the fmn mutant flies ( $N=444$ males observed) had a median survivorship of 18 days when aged on the same media (Table 3, Figure 3A). This reduction of six days represented a significant decrease in survivorship.

Table 3. Median day of survivorship and the total number of observed Drosophila deaths associated with DAT overexpression or loss of function.

\begin{tabular}{l|l|c|c}
\hline Genotype & Condition & Number of deaths observed & Median day of survivorship \\
\hline$w^{1118}$ & Control & 508 & 24 \\
\hline ; elav-Gal4/UAS-GFP & Control & 515 & 26 \\
\hline ; fmn & Mutant loss of function & 444 & 18 \\
\hline ; elav-Gal4/UAS-DAT & Over-expression & 463 & 20 \\
\hline
\end{tabular}

\section{A}

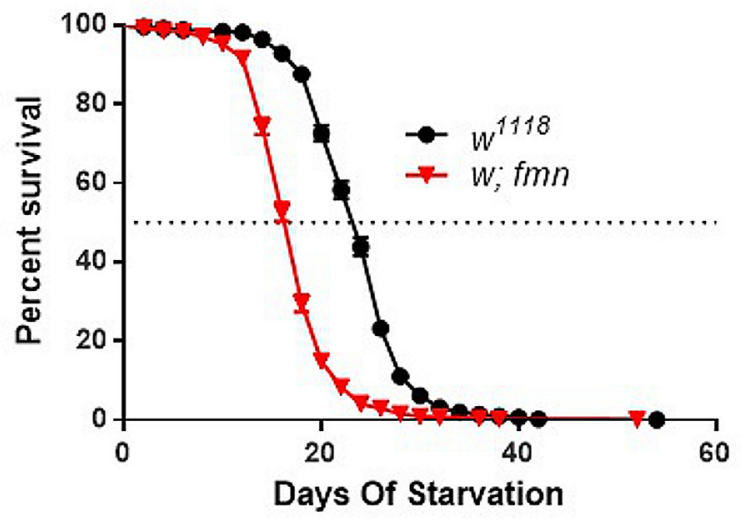

B

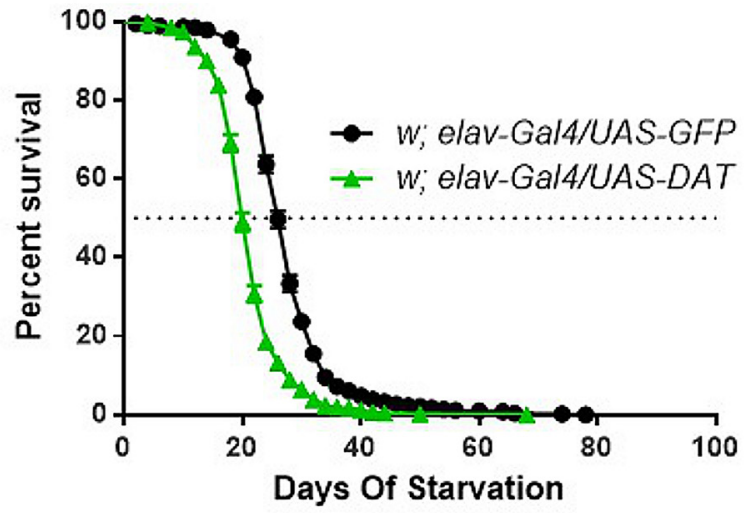

Figure 3. Drosophila survivorship is reduced with either excess or reduced DAT expression during amino acid starvation. A. Mutant loss of DAT function. Genotypes: $w^{1118}(\mathrm{~N}=508), w$; fmn $(\mathrm{N}=444)$. B. Overexpression of $D A T$. Genotypes: w; elav-Gal4/UAS-GFP $(\mathrm{N}=515)$, w; elav-Gal4/UAS-DAT $(\mathrm{N}=463)$. Survivorship is shown as percent survival $(P<0.05$ as determined by log rank). The dotted line represents the median survival of the flies. Error bars represent the standard error of the mean. 
Overexpression of DAT was achieved by the nervous system-specific driver elav-Gal4, which controlled the expression of UAS-DAT. The experimental control used the benign UAS-GFP, which was driven by elav-Gal4 ( $\mathrm{N}=515$ males observed), and control flies survived for a median of 26 days on the nutrient-deprived media (Table 3, Figure 3B). When DAT was overexpressed under the influence of elav-Gal4 ( $\mathrm{N}=463$ males observed), the median survival time was 20 days (Table 3 , Figure $3 \mathrm{~B}$ ). This difference of six days indicated a significant decline in survivorship.

\section{DISCUSSION}

The importance of a regulated internal mechanism, dependent upon neuropeptides and hormones, to control foraging and feeding in animals is evident. Here, we found that increases or decreases in the expression of the key neuropeptides NPF and SNPF and the neural hormone regulator DAT, could severely debilitate survivorship during conditions of nutritional adversity, particularly amino acid starvation.

NPF in insects is a structural homolog of the vertebrate NPY, which is considered to be the most powerful neuropeptide responsible for appetite enhancement (Valassi et al., 2008). In D. melanogaster, young larvae feed intensively to attain the weight required to undergo pupation. However, older larvae do not feed to the same extent, and they begin to wander before eventually finding a place to pupate (Melcher et al., 2007). In young larvae, NPF expression is upregulated, but there is less NPF expression in older larvae (Maule et al., 1995; de Jong-Brink et al., 2001). Young transgenic larvae that lack NPF signaling exhibit wandering phenotypes and a lack of feeding that is normally seen in older larvae (Wu et al., 2003). When larvae are starved they will "lower their standards" and eat foods that they might otherwise avoid, and they will forage when under suboptimal conditions (Wu et al., 2005, Lingo et al., 2007). Alteration of NPF expression affects these behaviors in that larvae with diminished NPF expression will continue to avoid noxious food even while being starved, and when the NPF receptor is impaired, fasted larvae will not forage. Certainly, a diminished appetite and a reduced amount of feeding could be expected to impair survivorship, especially under adverse nutrient conditions, and this was observed when NPF function was reduced in Drosophila that were aged on amino acid-depleted media.

Typically, NPF expression is lower in older larvae, but overexpression of NPF in these larvae leads to phenotypes similar to those observed in younger larvae. These phenotypes include increased feeding (Wu et al., 2003; Wu et al., 2005) that continues well past the normal developmental time-frame, resulting in delayed wandering and pupariation. Drosophila will not forage for food in colder conditions, but fasted larvae in a cold environment will forage when the expression of the NPF receptor is high (Lingo et al., 2007). Moreover, well-fed larvae with an overexpression of NPF will feed on noxious foods (Wu et al., 2005). While the amount of food ingested by Drosophila with overexpressed NPF was not measured in our experiments, the excess NPF expression and potential overfeeding did not increase survivorship of the flies on the amino acid-depleted media.

Variation of sNPF expression decreased the survivorship of Drosophila during the amino acid starvation assays. This shorter neuropeptide was implicated in the control of feeding, because the loss of expression in both larvae and adults resulted in organisms that did not feed; however, an abundance of sNPF expression in both larvae and adult Drosophila initiated feeding behavior (Lee et al., 2004). However, sNPF appears to function in the control of feeding in a different manner than NPF, particularly in larvae that have transitioned into the wandering stage. Both neuropeptides can 
either enhance (through overexpression) or inhibit (through loss of function) feeding in larvae prior to this stage. However, unlike the overexpression of NPF, alteration in sNPF expression does not enhance feeding beyond this point (Wu et al., 2003, Lee et al., 2004). Although it is not yet known if sNPF can affect feeding in malnourished larvae or adults in a way that is similar to NPF (Wu et al., 2005), our findings suggest that sNPF expression is pertinent to the survival of Drosophila exposed to depleted nutrients, because the elevation or reduction of sNPF limits the ability to survive under starvation conditions.

In vertebrates, feeding behavior may also be adjusted through the activity of the dopamine reward system, which is known to be activated post-ingestion. Feeding increases the release of dopamine, a response that becomes blunted in obese individuals. If tyrosine hydroxylase (TH), an enzyme necessary for the production of dopamine, is removed from dopaminergic neurons in mice, they do not feed (Zhou and Palmiter, 1995). The dopamine transporter functions to clear dopamine from the synapses, which reduces its expression and increases dopamine levels in the hypothalamus. Individuals suffering from eating disorders may undergo lengthy periods of dietary restriction, leading to lowered dopamine levels in the hypothalamus (Kontis and Theochari, 2012). Compared to free-fed animals, animals that have been feed-restricted typically show lesser expression of dopamine transporter (Bello et al., 2003). When DAT is actively reducing the levels of dopamine in the brain, hedonic feeding (feeding after being sated on sweet or high-fat foods) is reduced (Mebel et al., 2012). The dopamine levels of mice with a $10 \%$ reduction of DAT activity are approximately $70 \%$ higher compared to those of normal mice (Pecina et al., 2003). Moreover, these mice ate roughly $21 \%$ more, drank $15 \%$ more, gained roughly $5 \%$ more body weight, and worked harder for a sweet reward. Upon sucrose consumption after fasting, an exaggerated release of dopamine was observed (Polivy and Herman, 1985; Hagan and Moss, 1997; Bello et al., 2003). Given this information, Drosophila with impaired DAT function are expected to experience higher levels of dopamine signaling. Furthermore, these flies should eat more, while Drosophila that overexpress DAT should eat less. This may affect energy store levels and their ability to survive amino acid starvation. In our study, both instances led to increased sensitivity and decreased survivorship.

The majority of studies that examined the regulation of feeding behavior involved the insulin receptor signaling (IRS) pathway, which is well conserved between mammals and Drosophila (Britton et al., 2002). During conditions of low nutrient availability (e.g., amino acid starvation) the IRS signaling pathway is muted. With less insulin, more trehalose is present in the hemolymph, and this is coupled with an increase in lifespan (Broughton et al., 2008). The transcription factor foxo, a downstream effector that is negatively regulated by insulin receptor activity, is more active when IRS decreases. Moreover, foxo is important to survival during amino acid starvation (Kramer et al., 2008). Drosophila exposed to amino acid starvation media showed an increase in foxo expression, while the loss of foxo activity resulted in decreased survivorship when flies were only fed sucrose without protein or amino acids. The neuropeptide regulation of feeding is associated with this mechanism, because the cell surface of specific Drosophila neurons contains both the NPF receptor and the insulin receptor (Wu et al., 2005). When IRS is lowered in these neurons, larvae will overeat (including foods they would normally avoid). However, when there was an increase in IRS in these neurons, the larvae eat far less than expected. This suggests that the IRS negatively regulates the action of the NPF receptor, and thus NPF signaling in Drosophila. Therefore, one would expect that lowered NPF expression should share a similar outcome to what is observed with increased IRS during amino acid starvation and vice versa. However, our study reveals that 
both overexpression and impaired function of NPF results in sensitivity to amino acid starvation, which suggests that the mechanism is more complex than originally hypothesized.

In D. melanogaster, sNPF has been linked to IRS, because the neurons that express sNPF are in close proximity to insulin producing cells (Lee et al., 2008). Characterization of Drosophila with alterations to sNPF expression revealed that the phenotype of these flies was similar to that of flies with abnormal IRS levels. Hypomorphs of sNPF are $23 \%$ smaller than are controls, and they express decreased levels of the Drosophila insulin-like peptide 2 (Dilp2). Moreover, sNPF mutants exhibit downregulated $A k t 1$, increased levels of foxo located within the nucleus, increased 4EBP expression, and increased lifespans (Lee et al., 2008), and these results suggest that SNPF negatively regulates IRS. Consequently, lowering SNPF should increase foxo activity, which should then enhance survival during nutrient-deprived conditions. However, we found that a decrease in SNPF function did not enhance survival during amino acid starvation. Alternatively, sNPF overexpression led to $24 \%$ larger flies that showed an increase in Dilp2 expression (Lee et al., 2008). An increase in SNPF expression and binding to the sNPF receptor activates the IRS, and it supplies Dilp2 downstream of sNPF signaling. This increase in IRS should increase Akt1 activity and decrease foxo transcriptional activity, which should then sensitize the flies to amino acid starvation, and these results were observed in this study.

Dopamine signaling, strongly influenced by DAT activity, is linked to IRS, because the insulin receptor is expressed in the dopaminergic neurons of the midbrain of Drosophila (Palmiter, 2007; Mebel et al., 2012). Inhibition of the receptor reduces IRS and DAT expression; hence, dopamine clearance and the administration of insulin directly to the rat brain (via intracerebroventricular administration) lead to increases in DAT mRNA and activity that clear the synapses and decrease dopamine levels (Figlewicz et al., 1994). Rats fed a high-fat diet have decreased levels of Akt1 activity in the brain, and they require a longer period to clear the synapses of dopamine (Speed et al., 2011). Upon addition of the insulin receptor substrate, Akt1 levels are restored, and expression of DAT on the cell surfaces in the striatum is also reestablished. Other hormones that homeostatically control feeding behavior have also been shown to interact with receptors on neurons that secrete dopamine. Leptin, an anorexigenic hormone, inhibits dopamine activity, while ghrelin (an orexin) stimulates dopamine activity to influence the reward potential of feeding (Palmiter, 2007). Since lowered IRS and increased foxo activity are pertinent to the maximized survival of Drosophila undergoing amino acid starvation (Kramer et al., 2008), it would be expected that reduced DAT would result in the same phenotype, while overexpression of DAT would impair survival. However, we found that both upregulation and reduced activity of DAT decreased survivorship when flies were starved of amino acids.

It is clear the manipulation of components associated with the homeostatic and rewardbased control of feeding behaviors (e.g., the principal neuropeptides NPF and sNPF and the dopamine regulator DAT) affect survival during adverse nutrient conditions. While it is expected that overexpression should yield opposite results compared to lowering or loss of function experiments, this was not observed. These results indicated that it is important to maintain a sensitive balance in order to appropriately regulate the complex behavior of food intake, so that survivorship during adverse nutrient conditions can be maximized.

\section{Conflicts of interest}

The authors declare no conflicts of interest. 


\section{ACKNOWLEDGMENTS}

The authors would like to thank Dr. P. Shen (University of Georgia), Dr. K. Yu (Korea Research Institute of Bioscience and Biotechnology), Dr. R. Jackson (Tufts University), and the Bloomington Drosophila Stock Center (University of Indiana, Bloomington, USA) for the Drosophila lines. We thank Frankie Amanda Slade for helpful comments on the manuscript. JDS was funded by a Natural Sciences and Engineering Research Council of Canada Post-Graduate Scholarship and the School of Graduate Studies of Memorial University of Newfoundland. This project was funded by a Natural Sciences and Engineering Research Council of Canada Discovery Grant to BES.

\section{REFERENCES}

Arora S and Anubhuti (2006). Role of neuropeptides in appetite regulation and obesity--a review. Neuropeptides 40: $375-401$. http://dx.doi.org/10.1016/j.npep.2006.07.001

Bello NT, Sweigart KL, Lakoski JM, Norgren R, et al. (2003). Restricted feeding with scheduled sucrose access results in an upregulation of the rat dopamine transporter. Am. J. Physiol. Regul. Integr. Comp. Physiol. 284: R1260-R1268.http:// dx.doi.org/10.1152/ajpregu.00716.2002

Britton JS, Lockwood WK, Li L, Cohen SM, et al. (2002). Drosophila's insulin/PI3-kinase pathway coordinates cellular metabolism with nutritional conditions. Dev. Cell 2: 239-249.http://dx.doi.org/10.1016/S1534-5807(02)00117-X

Broughton S, Alic N, Slack C, Bass T, et al. (2008). Reduction of DILP2 in Drosophila triages a metabolic phenotype from lifespan revealing redundancy and compensation among DILPs. PLoS One 3: e3721.http://dx.doi.org/10.1371/journal. pone.0003721

Cagniard B, Balsam PD, Brunner D and Zhuang X (2006). Mice with chronically elevated dopamine exhibit enhanced motivation, but not learning, for a food reward. Neuropsychopharmacology 31: 1362-1370.http://dx.doi.org/10.1038/sj.npp.1300966

Cannon CM, Abdallah L, Tecott LH, During MJ, et al. (2004). Dysregulation of striatal dopamine signaling by amphetamine inhibits feeding by hungry mice. Neuron 44: 509-520.http://dx.doi.org/10.1016/j.neuron.2004.10.009

de Bono $\mathrm{M}$ and Bargmann $\mathrm{Cl}$ (1998). Natural variation in a neuropeptide $\mathrm{Y}$ receptor homolog modifies social behavior and food response in C. elegans. Cell 94: 679-689.http://dx.doi.org/10.1016/S0092-8674(00)81609-8

de Jong-Brink M, ter Maat A and Tensen CP (2001). NPY in invertebrates: molecular answers to altered functions during evolution. Peptides 22: 309-315.http://dx.doi.org/10.1016/S0196-9781(01)00332-1

Fairburn CG and Harrison PJ (2003). Eating disorders. Lancet 361: 407-416.http://dx.doi.org/10.1016/S0140-6736(03)12378-1

Figlewicz DP, Szot P, Chavez M, Woods SC, et al. (1994). Intraventricular insulin increases dopamine transporter mRNA in rat VTA/substantia nigra. Brain Res. 644: 331-334.http://dx.doi.org/10.1016/0006-8993(94)91698-5

Garczynski SF, Brown MR, Shen P, Murray TF, et al. (2002). Characterization of a functional neuropeptide F receptor from Drosophila melanogaster. Peptides 23: 773-780.http://dx.doi.org/10.1016/S0196-9781(01)00647-7

Hagan MM and Moss DE (1997). Persistence of binge-eating patterns after a history of restriction with intermittent bouts of refeeding on palatable food in rats: implications for bulimia nervosa. Int. J. Eat. Disord. 22: 411-420.http://dx.doi. org/10.1002/(SICl)1098-108X(199712)22:4<411::AID-EAT6>3.0.CO;2-P

Hoebel BG (1971). Feeding: neural control of intake. Annu. Rev. Physiol. 33: 533-568.http://dx.doi.org/10.1146/annurev. ph.33.030171.002533

Kontis D and Theochari E (2012). Dopamine in anorexia nervosa: a systematic review. Behav. Pharmacol. 23: 496-515.http:// dx.doi.org/10.1097/FBP.0b013e328357e115

Kramer JM, Slade JD and Staveley BE (2008). foxo is required for resistance to amino acid starvation in Drosophila. Genome 51: 668-672.http://dx.doi.org/10.1139/G08-047

Kume K, Kume S, Park SK, Hirsh J, et al. (2005). Dopamine is a regulator of arousal in the fruit fly. J. Neurosci. 25: $7377-7384$. http://dx.doi.org/10.1523/JNEUROSCl.2048-05.2005

Lee KS, You KH, Choo JK, Han YM, et al. (2004). Drosophila short neuropeptide F regulates food intake and body size. J. Biol. Chem. 279: 50781-50789.http://dx.doi.org/10.1074/jbc.M407842200

Lee KS, Kwon OY, Lee JH, Kwon K, et al. (2008). Drosophila short neuropeptide F signalling regulates growth by ERKmediated insulin signalling. Nat. Cell Biol. 10: 468-475.http://dx.doi.org/10.1038/ncb1710

Lingo PR, Zhao Z and Shen P (2007). Co-regulation of cold-resistant food acquisition by insulin- and neuropeptide Y-like systems in Drosophila melanogaster. Neuroscience 148: 371-374.http://dx.doi.org/10.1016/j.neuroscience.2007.06.010

Maule AG, Shaw C, Halton DW, Thim L, et al. (1991). Neuropeptide F: a novel parasitic flatworm regulatory peptide from 
Moniezia expansa (Cestoda: Cyclophyllidea). Parasitology 102: 309-316. http://dx.doi.org/10.1017/S0031182000062648

Maule AG, Halton DW and Shaw C (1995). Neuropeptide F: a ubiquitous invertebrate neuromediator? Hydrobiologia 305: 297303. http://dx.doi.org/10.1007/BF00036410

Mebel DM, Wong JC, Dong YJ and Borgland SL (2012). Insulin in the ventral tegmental area reduces hedonic feeding and suppresses dopamine concentration via increased reuptake. Eur. J. Neurosci. 36: 2336-2346.http://dx.doi.org/10.1111/ j.1460-9568.2012.08168.x

Melcher C, Bader R and Pankratz MJ (2007). Amino acids, taste circuits, and feeding behavior in Drosophila: towards understanding the psychology of feeding in flies and man. J. Endocrinol. 192: 467-472.http://dx.doi.org/10.1677/JOE$\underline{06-0066}$

Nässel DR and Wegener C (2011). A comparative review of short and long neuropeptide F signaling in invertebrates: Any similarities to vertebrate neuropeptide $Y$ signaling? Peptides 32: 1335-1355.http://dx.doi.org/10.1016/j. peptides.2011.03.013

Nielsen S (2001). Epidemiology and mortality of eating disorders. Psychiatr. Clin. North Am. 24: 201-214, vii-viii.http://dx.doi. org/10.1016/S0193-953X(05)70217-3

Owusu-Ansah E and Perrimon N (2014). Modeling metabolic homeostasis and nutrient sensing in Drosophila: implications for aging and metabolic diseases. Dis. Model. Mech. 7: 343-350.http://dx.doi.org/10.1242/dmm.012989

Palmiter RD (2007). Is dopamine a physiologically relevant mediator of feeding behavior? Trends Neurosci. 30: 375-381. http:// dx.doi.org/10.1016/j.tins.2007.06.004

Peciña S, Cagniard B, Berridge KC, Aldridge JW, et al. (2003). Hyperdopaminergic mutant mice have higher "wanting" but not "liking" for sweet rewards. J. Neurosci. 23: 9395-9402.

Polivy $\mathrm{J}$ and Herman CP (1985). Dieting and binging. A causal analysis. Am. Psychol. 40: 193-201.http://dx.doi. org/10.1037/0003-066X.40.2.193

Schultz W (2006). Behavioral theories and the neurophysiology of reward. Annu. Rev. Psychol. 57: 87-115.http://dx.doi. org/10.1146/annurev.psych.56.091103.070229

Sotak BN, Hnasko TS, Robinson S, Kremer EJ, et al. (2005). Dysregulation of dopamine signaling in the dorsal striatum inhibits feeding. Brain Res. 1061: 88-96.http://dx.doi.org/10.1016/j.brainres.2005.08.053

Speed N, Saunders C, Davis AR, Owens WA, et al. (2011). Impaired striatal Akt signaling disrupts dopamine homeostasis and increases feeding. PLoS One 6: e25169.http://dx.doi.org/10.1371/journal.pone.0025169

Valassi E, Scacchi M and Cavagnini F (2008). Neuroendocrine control of food intake. Nutr. Metab. Cardiovasc. Dis. 18: 158168.http://dx.doi.org/10.1016/j.numecd.2007.06.004

Vanden Broeck J (2001). Neuropeptides and their precursors in the fruitfly, Drosophila melanogaster. Peptides 22: 241-254. http://dx.doi.org/10.1016/S0196-9781(00)00376-4

Wu Q, Wen T, Lee G, Park JH, et al. (2003). Developmental control of foraging and social behavior by the Drosophila neuropeptide Y-like system. Neuron 39: 147-161.http://dx.doi.org/10.1016/S0896-6273(03)00396-9

Wu Q, Zhao Z and Shen P (2005). Regulation of aversion to noxious food by Drosophila neuropeptide $Y$ - and insulin-like systems. Nat. Neurosci. 8: 1350-1355.http://dx.doi.org/10.1038/nn1540

Yamamoto S and Seto ES (2014). Dopamine dynamics and signaling in Drosophila: an overview of genes, drugs and behavioral paradigms. Exp. Anim. 63: 107-119.http://dx.doi.org/10.1538/expanim.63.107

Zhou Q-Y and Palmiter RD (1995). Dopamine-deficient mice are severely hypoactive, adipsic, and aphagic. Cell 83: 11971209.http://dx.doi.org/10.1016/0092-8674(95)90145-0 